\title{
Study of removal of direct black ANBN dye from aqueous solution by chitosan-functionalized mesoporous (SBA-15) composites synthesized by electrospinning method
}

\author{
Masoudnia S., Juybari M.H.*, Mehrabian R.Z., Ebadi M. and Kaveh F. \\ Department of Chemistry, Faculty of Science, Gorgan Branch, Islamic Azad University, Gorgan, Iran \\ Received: 04/10/2019, Accepted: 19/10/2020, Available online: 09/11/2020 \\ *to whom all correspondence should be addressed: e-mail: m.habibi@gorganiau.ac.ir
}

https://doi.org/10.30955/gnj.003228

\section{Graphical abstract}
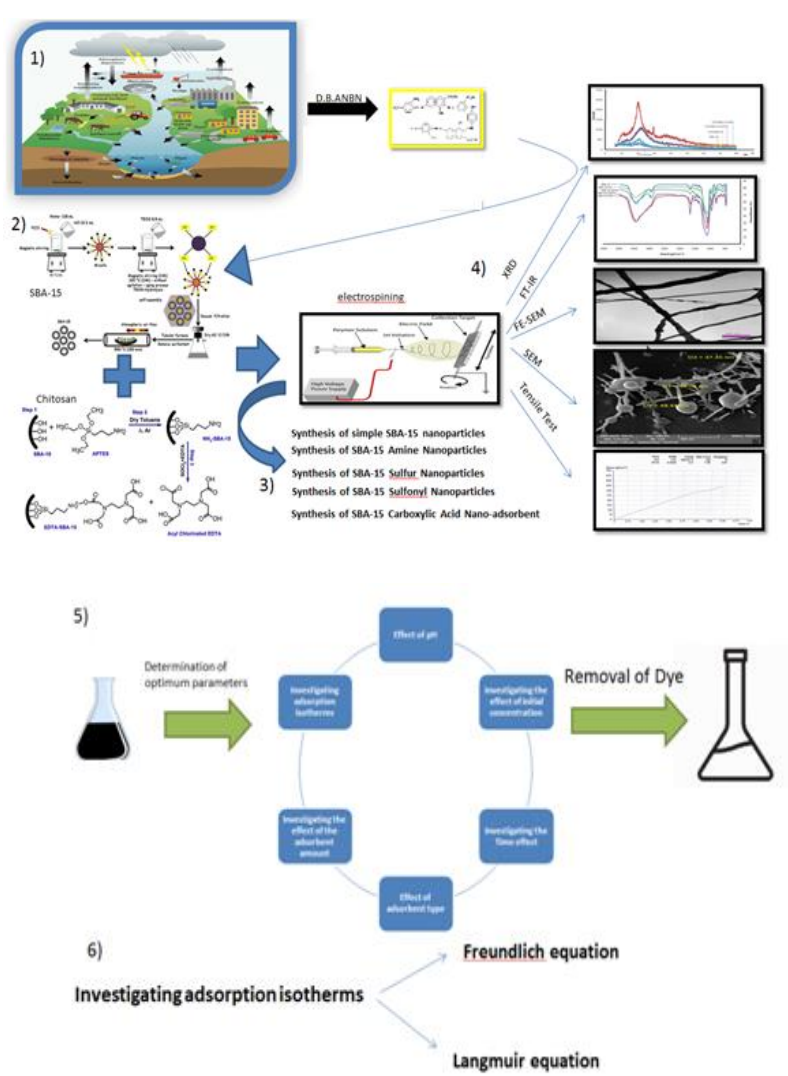

Abstract
In this research, capability of adsorption of modified
chitosan Nano composites with functionalized mesoporous
silica which synthesized by electrospinning method in the
removal of direct black ANBN dye has been studied. Silicate
mesoporous were functionalized during synthesis process
and then the intended Nano fibers were produced by using
of electrospinning device. Synthesized Nano fibers were
recognized by analyzing the FT-IR, XRD, FE-SEM, and TEM
and tension test. The removal of direct black ANBN dye
from aqueous solution in a continuous system was done by
this adsorbent by studying the effects of some parameters such as type and amount of adsorbent, $\mathrm{pH}$, initial concentration of colored solution and contact time; And at the end finding appropriate isotherms for direct black dye adsorption was done. The result show that simple CTS/SBA15 have the best removal efficiency in $40 \mathrm{~min}$ of contact time, in acidic $\mathrm{pH}$, with $0.05 \mathrm{gr}$ adsorbent and $60 \mathrm{mg} / \mathrm{L}$ initial concentration. By examining the adsorption isotherms, it has been clearly defined [that] Removal of this dye follows Freundlich model. In order to compare accurately and evaluate the efficiency of the synthesized sample, the adsorption of Direct Black ANBN was investigated by two different adsorbents: CTS/SBA-15 and Chitosan. According to the results, CTS/SBA-15 Nano fibers have suitable efficiency for removal of direct black ANBN dye.

Keywords: Removal, dye, Nano composite, mesoporous silica, functionalized, electrospinning

\section{Introduction}

One of major sources of environmental pollution is industrial wastewater released into the water bodies (Kaur et al., 2010). The discharge of wastes into the water bodies had brought change of the environmental water quality; hence making substantial quantities of water is unsuitable for various uses. One of environmental issues for many countries especially developing nations is compromise in the quality of the environment as a result of effluent discharge from the industrial sectors (Ohima et al., 2009). A remarkable impact on the receiving water bodies is created by release of this industrial wastewater into the environment. This is especially true for chemical and similar process industries like the paint industry. International Union Conservation Nature and Natural Resources (IUCN) determine $75 \mathrm{mg} / \mathrm{l}$ for wastewater disposal, the discharge limit of dye into surface water, sewage wells, agricultural, and irrigation uses. Dyes which are widely used in different industries such as paper, plastic, textile, rubber and mostly produce severe environmental pollution, in the form of colored wastewater excreted into waters of environment (Jing et al., 2008). More than 7000000 tons out of almost 10000 kind of dyes and pigments are produced in all over 
the world every year that nearly $20 \%$ of this amount are discharged as industrial wastewater during dying and complementary process without initial preparation (Khataee et al., 2011; Yue et al., 2010). Most of dyes are stable, and not easily degradable by the conventional treatment methods, so removal of dyes from the textile effluents is a major problem (Uysal et al., 2018). Synthetic dyes are used in various industrial dyeing and printing processes. Textile industry is the largest user of synthetic dyes (Sarioglu et al., 2017). Among these dyes, Azo dyes are included almost $70 \%$ of dyes available in the world (GarciaSegura et al., 2013). The color and toxicity of dyes cause some issues for people's health by affecting the efficiency of water filtration techniques which effect on the quality of life (Munusamy et al., 2015; Nunes et al., 2007). Colored pollutants are important and easy to be observed even in amounts less than $1 \mathrm{mg} / \mathrm{L}$ (Nilsson et al., 2006), this is why removal of them from aqueous solutions is required. Since filtering of colored wastewater by common biologically and physicochemical process is relatively done with difficulties because of their chemical complex structure; adsorption process has been utilized as an effective alternative to filter the colored waste water and remove toxic compound from industrial wastewater (Nilsson et al., 2006). Among adsorbents used, activated Carbon is one of the most constructive and widest adsorbents which is used. However, because it's initial substances are not native, [activated Carbon] is relatively expensive, has high running costs and regenerating that has numerous problems (Liversidge et al., 1997). Searching for low cost, accessible, simple implementation, and environmentally friendly adsorbents has been one of the concern of researchers in recent years (Shokoohi et al., 2018) There for, the need of available adsorbents with low expense seems to be necessary SBA-15 is one of the most popular mesoporous silicate which was presented by Zhao and his co-workers in Santa Barbara area, California in 1998 (Zhao et al., 1998). Special characteristics such as high contact surface, high porosity, adjustable and small pores size, regular and uniform distribution cause the popularity of this mesopour silicate (Da'na et al., 2011). Designing adsorbents with meso structure for removal of different dyes from aqueous solutions is one of the newfound issues in the filtration of water and industrial waste waters field (Burleigh et al., 2001). By placing different functional groups on the surface of adsorbent channels can take a step toward modifying the structure and their cavity size which causes more various and more practical adsorbents to be produced (Badiee et al., 2006).

At present, Nano fibers production has attracted the attention of researchers and scientists because of Nanometer dimensions and their physical, chemical and biological features. When diameter of Nano fibers decreases to Nanometer from micrometer, special characteristics such as great special surface and high mechanical efficiency appears; this, has caused them to be used in so many of recent research (Bahrambeigi et al., 2013; Nasouri et al., 2012). There are several ways to synthesize the Nano fibers [that] electrospinning (A simple and an effective method for producing optimal adsorbent with high a/v and high porosity) has been known as a beneficial and successful method among them. Different substances such as polymers can be transformed into fibers in Nano to micro range by this method (An et al., 2017). Nowadays, Nano fibers are utilized in various fields like environmental engineering, biotechnology, defense and military industries, electronic, filtration and.... (Nasouri et al., 2012; Rabbi et al., 2012; Saeed et al., 2012).

In this research, the aim is to take a step toward using these adsorbents in wide range as a placement for commercial expensive adsorbents; by examining the efficiency of chitosan-functionalized mesoporous (SBA-15) composites in removal of direct black ANBN dye.

\section{Materials and methods}

\subsection{Chemical materials and devices}

Direct black ANBN dye was bought from Haft Rang Company in Iran. All the chemical substances were prepared from Merck Company in Germany and Sigma Aldrich Company in America. All the materials which were utilized in this research had laboratorial degree.

In order to identify and recognize all functional groups placed on the surface of the nanofibers synthesized, the Fourier Spectrometer Model VERTEX 70 of Broker Company in Germany was used. FE-SEM field emission electron model MIRA3TESCAN-XMU was used to determine the surface morphology and appearance of nanofibers. The hexagonal structure of the synthesized adsorbents was observed with the TEM image of Technai G5 transmission electron microscope model Leo 1455VP at $300 \mathrm{kV}$. The XRD spectra were prepared by using of Philips $X^{\prime}$ pert device with radiation source of $\mathrm{Cu}-\mathrm{K} \alpha$ and the spectroscopy was carried out in the order of 20(2Theta(degree)). Tensile testing was performed to determine the tensile strength of synthesized nanofibers by using of tensile device model Tinif-olsen $\mathrm{H} 10 \mathrm{KT}$ made in the United Kingdom with the use of Q-Mat software. To determine the residual concentration of dyes, UV-Visible spectrophotometers model DR500 of HATCH company in America was used.

\subsection{Synthesis of SBA-15 nanoadsorbents}

\subsubsection{Synthesis of simple SBA-15 nanoadsorbents}

SBA-15 nanoadsorbent was synthesized in the way that Zhao and his co-workers reported (Zhao et al., 1998). For this aim, $2 \mathrm{gr} \mathrm{P}-123$ surfactant was stirred in $62.5 \mathrm{gr} \mathrm{HCl} 1.9$ $\mathrm{M}$ at $45^{\circ} \mathrm{C}$ for $45 \mathrm{~min}$. After this period of time, $3.84 \mathrm{gr}$ Tetraethyl orthosilane (TEOS) was added to the mentioned mixture and were stirred for $45 \mathrm{~min}$ at previous temperature. Then by increasing the temperature to $100^{\circ} \mathrm{C}$ of, the reaction mixture was put for rest for $24 \mathrm{hrs}$. After passing the time mentioned, the white solid substance was obtained. This white solid with $50 \mathrm{ml}$ ethanol were put in reflex situation for $24 \mathrm{hrs}$. at $78^{\circ} \mathrm{C}$. At the end, the reaction mixture was filtered and washed with a few amount of deionized water.

\subsubsection{Synthesis of amine SBA-15 nanoadsorbent}


In order to synthesis of amine SBA-15 nanoadsorbent corresponded to described method in previous essay (Boorboor Ajdari et al., 2016), 2 gr P-123 was dissolved in $62.5 \mathrm{gr} \mathrm{HCL} \mathrm{M}$ and the reaction mixture was heatedto $45^{\circ} \mathrm{C}$ and $3.84 \mathrm{gr}$ of TEOS was added to that and stirred by magnetic shaker for $45 \mathrm{~min}$ at previous temperature and then $1.0181 \mathrm{gr}$ of APTES (to make amine group) was added to that and was stirred for $24 \mathrm{hrs}$. at mentioned condition. Then, by increasing the temperature to $100^{\circ} \mathrm{C}$, the reaction mixture was put for rest. P-123 was removed under reflux condition by $50 \mathrm{ml}$ ethanol. The final production was filtered off with a filter paper and washed several times with distilled water.

\subsubsection{Synthesis of sulfur SBA-15 nanoadsorbent}

Synthesis of sulfur SBA-15 nanoadsorbent was performed similar the method reported in previous essays (Zhao et al., 2016). $2 \mathrm{gr}$ of $\mathrm{P}-123$ was dissolved in $62.5 \mathrm{gr}$ of $1.9 \mathrm{M} \mathrm{HCL}$ and it was heated to $40^{\circ} \mathrm{C}$ for $45 \mathrm{~min}$, then $3.84 \mathrm{gr}$ of TEOS was added to that and was stirred at previous temperature for 45 min one more time... 1.131 gr of MPTES (To form sulfur group) was added to that. Reaction mixture was stirred for $24 \mathrm{hrs}$ at $45^{\circ} \mathrm{C}$ after that by increasing the temperature to $100^{\circ} \mathrm{C}$, the reaction mixture was put to rest. P-123 was removed by ethanol under reflux condition for $24 \mathrm{hrs}$. The solid solution was filtered off with a filter paper and washed several times with distilled water.

\subsubsection{Synthesis of sulfonic SBA-15 nanoadsorbent}

First, $4 \mathrm{gr}$ of $\mathrm{P}-123$ was dissolved in $97.58 \mathrm{gr}$ deionized water for $2 \mathrm{hrs}$ at $45^{\circ} \mathrm{C}$, then $1.97 \mathrm{gr}$ of $\mathrm{HCL} 37 \%$ was added to that and again it was stirred for $45 \mathrm{~min}$. Next, $7.69 \mathrm{gr}$ of TEOS was added to the reaction mixture and was stirred for $2 \mathrm{hrs}$ at $45^{\circ} \mathrm{C}$. $0.81 \mathrm{gr}$ of MPTES with $8.37 \mathrm{gr}$ of $\mathrm{H}_{2} \mathrm{O}_{2}$ were added to reaction mixture and were stirred for $24 \mathrm{hrs}$. Then, by increasing the temperature to $100^{\circ} \mathrm{C}$, the reaction mixture was put in stable condition. $\mathrm{P}-123$ was removed by ethanol under reflux condition for $24 \mathrm{hrs}$. The solid solution was filtered off with a filter paper and washed several times with distilled water (Erdem et al., 2017).

\subsubsection{Synthesis of carboxylic acid nanoadsorbent}

$1 \mathrm{gr}$ of amine SBA-15 was poured in $25 \mathrm{ml}$ dry Dimethyl formamide (DMF). Then $0.2 \mathrm{gr}$ of succinic anhydride with $0.02 \mathrm{gr}$ of Dicyclohexylrcarbamide (DDC) was poured in the container containing $25 \mathrm{ml}$ DMF. Solution containing SBA15 , was added dropwise to the solution containing succinic anhydride which was being heavily stirred and then the solution was stirred for $24 \mathrm{hrs}$. The sample obtained was washed with DMF and ethanol and was dried in Suck sailed ethanol (Badiee et al., 2014).

\subsection{Preparing the solutions for electrospinning}

\subsubsection{Preparation of $0.5 \%$ SBA-15 nanoadsorbent mass solution}

$0.2 \mathrm{gr}$ of synthesized nanoadsorbent was added to $39.8 \mathrm{gr}$ of deionized water and was stirred for $10 \mathrm{~min}$ by ultrasonic device to form a completely uniform solution.

\subsubsection{Preparation of $3 \%$ chitosan mass solution}

$97 \mathrm{gr}$ acetic acid was added to 3 gr chitosan powder and after a few min stirring by glass mixture, there were stirred for $15 \mathrm{~min}$ by ultrasonic device.

\subsubsection{Preparation of simple and functionalized CTS/SBA-15 composite nanofibers}

For preparing nanofiber, $16 \mathrm{ml}$ of $0.5 \%$ SBA-15 solution was stirred with $64 \mathrm{ml} 3 \%$ chitosan solution and after a few min stirring with glass mixer, they were stirred by ultrasonic device for $20 \mathrm{~min}$ to obtain the jelly uniform solution. The solution obtained was moved to electrospinning device in order to synthesize the nanofibers and after $18 \mathrm{hrs}$, the desired nanocomposite was taken out from the device in the shape of porous narrow film.

\subsection{Condition of electrospinning device}

The desired nanofiber was synthesized with the use of an electrospinning device. The structure and method of operation of this device is based on a horizontal projection of the polymer solution in which the material is fed to a syringe using a pump located outside the cab. The electrospinning process is carried out between the needle tip, which is connected to the positive output of a high voltage source, and is coated with a copper sheet with an aluminum foil or Teflon sheet with graphite (Figure 1). All procedures were performed under the same conditions using an electric current by keeping the distance between the needle and collector at $20 \mathrm{~cm}$ and feed rate at 0.212 $\mathrm{ml} / \mathrm{h}$ and voltage change $(11.5,13,14.5 \mathrm{~V}$ ) (Almuhamed et al., 2014).

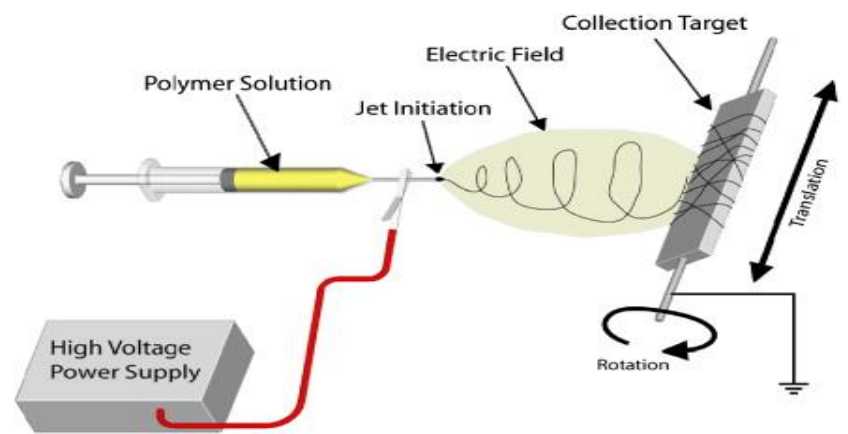

Figure 1. Schematic of electrospinning method

\subsection{Experiment of surface adsorption}

Adsorption experiment was performed by batch synthesized CTS/SBA-15 nanofibers. In order to perform the experiments, certain amounts of the synthesized nanofibers $(0.03 \mathrm{gr}$ ) were weighed firs, poured into $250 \mathrm{ml}$ Erlenmeyer flask and $50 \mathrm{ml}$ of dye solution was added to that. Subsequently, the desired solution was stirred on the shaker with a rotation speed of $100 \mathrm{rpm}$ and room temperature for $30 \mathrm{~min}$. At the specified times the solutions were removed from shaker, the adsorbent was separated and the residual dye concentration in the solution was determined by UV-Vis spectrophotometer.

Equation 1 was used to determine the percentage of dye removal: 


$$
\operatorname{Removal}(\%)=\frac{A_{0}-A_{t}}{A_{0}} \times 100
$$

In this equation $A_{0}$ and $A_{t}$ are adsorption at zero and $t$ moment, respectively.

The amount of dye adsorbed $\left(q_{t}\right)$ on the adsorbent surface was calculated from the equation 2 in terms of $\mathrm{mg} / \mathrm{g}$ adsorbent at the zero and t moments:

$$
\mathrm{q}_{\mathrm{t}}=\left(\frac{\mathrm{C}_{0}-\mathrm{C}_{\mathrm{t}}}{\mathrm{m}}\right) \times \mathrm{V}
$$

Where $\mathrm{C}_{0}$ is the dye concentration at moment zero, $\mathrm{C}_{\mathrm{t}}$ is the dye concentration at moment $t, m$ is the adsorbent mass and $V$ is the volume of solution.

Table 1. FT-IR vibration of functional groups for simple and functionalized CTS/SBA-15 nanofibers.

\begin{tabular}{cc}
\hline Frequency $(\mathbf{c m}-\mathbf{1})$ & Bond \\
\hline 811 & Si-O-Si \\
\hline 1068 & Si-O-Si \\
\hline 1570 & $\mathrm{~N}-\mathrm{H}$ \\
\hline $1600-1800$ & $\mathrm{C}=\mathrm{O}$ \\
\hline 2570 & $\mathrm{~S}-\mathrm{H}$ \\
\hline $3000<$ & $\mathrm{O}-\mathrm{H}$ \\
\hline 3745 & $\mathrm{Si}-\mathrm{OH}$ \\
\hline
\end{tabular}

\section{Result and discussion}

\subsection{FT-IR result}

The result of FT-IR vibration of all the synthesized nanofibers could be observed in Table 1 and Figure 2 . The peak observed at $811 \mathrm{~cm}^{-1}$ and $1068 \mathrm{~cm}^{-1}$ corresponded to the symmetric and asymmetric stretching vibration of the $\mathrm{Si}-\mathrm{O}-\mathrm{Si}$ bond in the silicate compressed interconnected network, respectively. The sharp peak in the $3745 \mathrm{~cm}^{-1}$ area is ascribed to the $\mathrm{Si}-\mathrm{OH}$ silane groups. Bond adsorbed at $1570 \mathrm{~cm}^{-1}$ is ascribed to the bending vibration of amine group, peak observed at $2570 \mathrm{~cm}^{-1}$ is ascribed to the vibration of $\mathrm{S}-\mathrm{H}$ group and the peaks appeared in the range of $1600 \mathrm{~cm}^{-1}-1800 \mathrm{~cm}^{-1}$ are attributed to the bending vibration of $\mathrm{C}=\mathrm{O}$ bonds. Stretching vibration of $\mathrm{OH}$ was appeared above $3000 \mathrm{~cm}^{-1}$. Based on the results obtained from FT-IR analysis it can be stated that, the desired organic functional groups were placed on the surface of silica mesopours nano pores successfully.

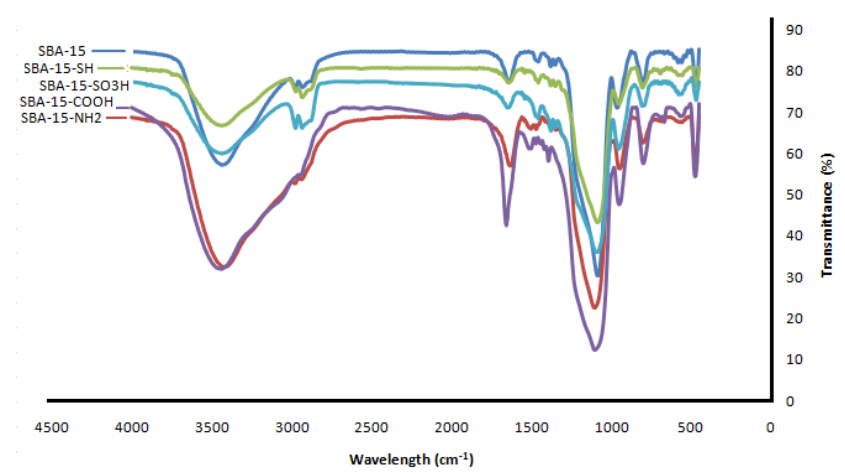

Figure 2. Comparison of SBA-15, SBA-15- $\mathrm{NH}_{2}$, SBA-15-SH, SBA$15-\mathrm{SO}_{3} \mathrm{H}, \mathrm{SBA}-15-\mathrm{COOH}$ of FT-IR

\subsection{Results of FE-SEM}

FE-SEM image of synthesized nanofibers in different size of magnifications has been shown in Figure 3. A regular and tidy structure with relatively uniform size is observed in FESEM images. FE-SEM images also show that SBA-15 nanocomposite is a hexagonal structure with almost uniform sizes. In addition to that, polymerization of agglomeratesalso shows the symmetry of hexagonal in silicate mesopours. Morphologically, the sample consists of a large number of cluster units of relatively uniform size and a large number of string assemblies, which appear to be perfectly consistent with the results of previous valid studies. The slight variation in the diffraction angles in this study from previous studies may be due to the difference in the synthesis techniques and the calcination temperature (Ullah et al., 2015).
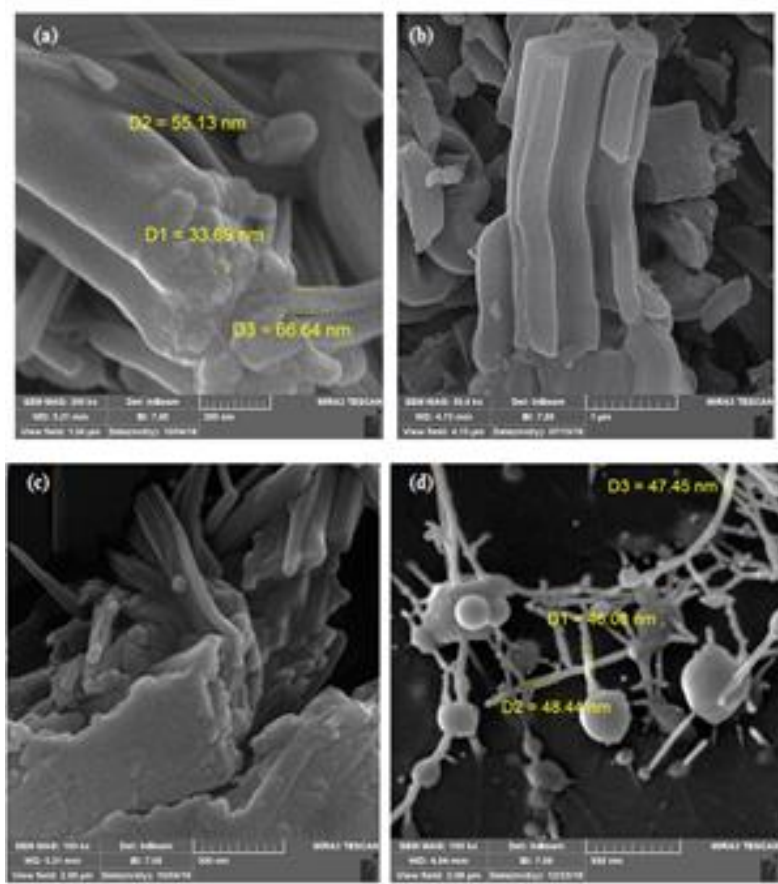

Figure 3. FT-IR image of, a, b, c: SBA-15, d: CTS/SBA-15

\subsection{TEM results}

Morphological analysis of the synthesis of CTS/SBA-15 nanofibers by TEM has been shown in Figure 4 . As can be seen, the TEM images of the cylindrical hollow channels and the highly ordered hexagonal arrangement with high uniformity and the size of the cavities in the form of parallel and tubular walls can be clearly seen as a common feature of the SBA-15 compounds. The structure of the synthesized compounds is also visible in the honeycomb hexagon. The TEM results confirmed that the modification occurred within the pores and no structural deformation was observed in the SBA-15 structure after the formation of nanocomposites (Devaraju et al., 2013)

\section{4. $X R D$ results}

Figure 5 shows the XRD pattern of the synthesized compounds. The XRD pattern for SBA-15 has four significant peaks. Peaks can be indexed as (100), (110), (200) and (210) and are associated with hexagonal 
symmetry. The XRD pattern shows that SBA-15 has single hexagonal arrays. The peaks corresponding to the crystal plates 100, 110 and 210 indicate the formation of a highly ordered hexagonal structure in the sample, and peak 100 indicates the cavity order in the nanofibers structure (Alexa et al., 2012).

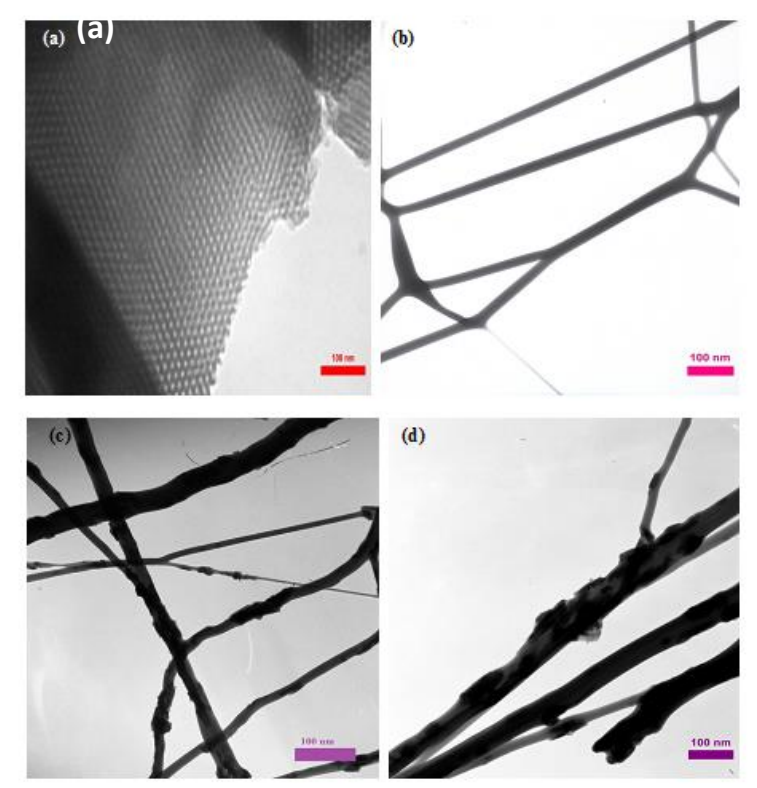

Figure 4. TEM image of a: SBA-15, b: CTS, c, d: CTS/SBA-15-NH2

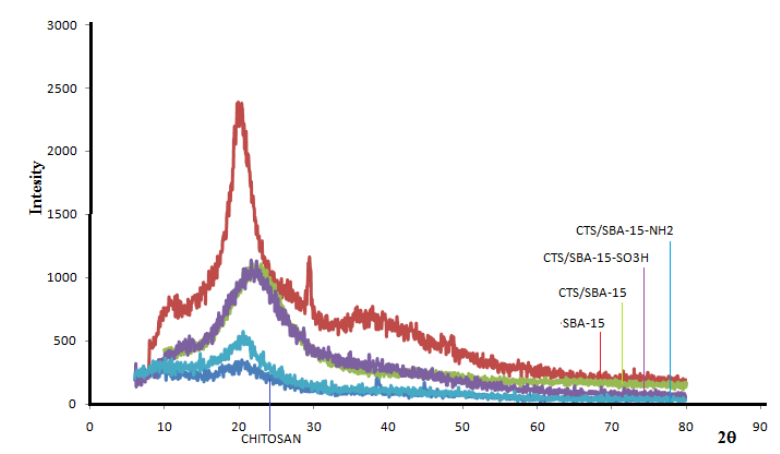

Figure 5. Comparison of XRD chitosan pattern, CTS/SBA-15$\mathrm{SO}_{3} \mathrm{H}, \mathrm{CTS} / \mathrm{SBA}-15, \mathrm{SBA}-15, \mathrm{CTS} / \mathrm{SBA}-15-\mathrm{NH}_{2}$

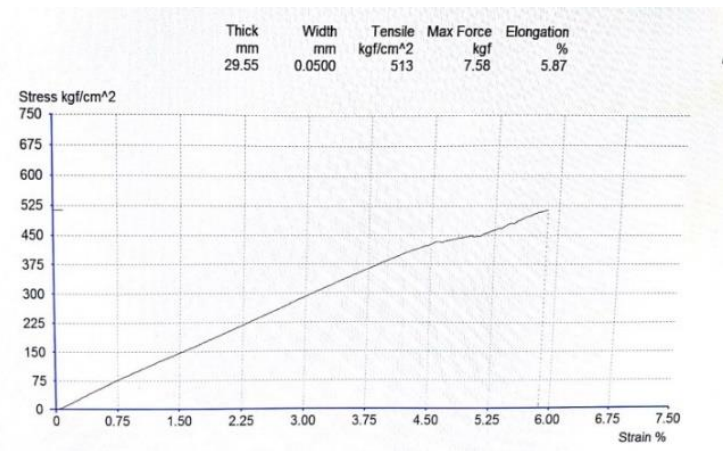

Figure 6. Stress Test Strain Test Diagram

\subsection{Tensile test result}

Tensile testing is one of the most important mechanical properties tests. To perform this test, the sample must first be duplicated in standard size, then the sample is inserted between the two jaws, and the device begins to draw at standard speed, with simultaneous power and resistance values recorded by the computer. As the sample dimensions are accurately measured with the caliper, the stress is obtained by dividing the force on the surface, and by dividing the displacement on the initial length, strain is obtained. And the strain-stress curve can be plotted. According to the strain-stress curve, the behavior of the material determines whether it is brittle or hard (Figure 6).

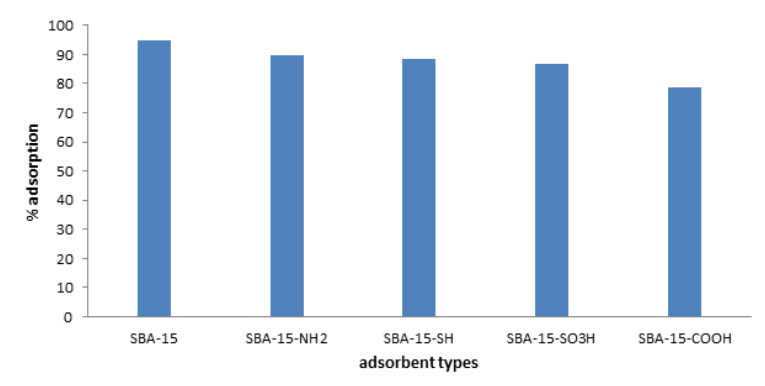

Figure 7. Effect of adsorbent types on the adsorption of Direct Black (0.03gr adsorbent, $\mathrm{pH}=7$, initial concentration: $20 \mathrm{mg} / \mathrm{l}, 30$ min of time contact)

The synthesized nanofibers are so strong that they can withstand much stress without deformation, according to the above diagram, the results show that the mentioned material can withstand a lot of stress and strain and this rate is improved and increased due to the functionalization of the synthesized compounds.

\subsection{Effect of type of adsorbent}

To investigate the effect of the type of nanofibers synthesized by the electrospinning process on removal of direct black ANBN dye, $0.03 \mathrm{gr}$ of simple, amine, sulfur, sulfonic and carboxylic acid CTS/SBA-15 nanofibers were used for the desired dye solution at concentration of 20 $\mathrm{mg} / \mathrm{l}$. in each experiment, the adsorbent sample was contacted with $50 \mathrm{ml}$ of dye solution for $30 \mathrm{~min}$. Results have been presented in Figure 7. Based on the results, simple CTS/SBA-15 nanofibers selected as the best adsorbent because of their better adsorption sites and more efficient adsorption characteristics than other nanofibers.

\subsection{Effect of $p H$}

$\mathrm{pH}$ is the most important parameter which has an effect on the adsorption process. In this research, effect of $\mathrm{pH}$ on the adsorption of direct black ANBN dye was studied in acidic, basic and neutral ph.In order to examine the influence of $\mathrm{pH}, \mathrm{HCl}$ and $\mathrm{NaOH}$ solutions were adjusted with $\mathrm{pH}$ in the range of 2-11 by the use of $\mathrm{pH}$ meter.At this stage, $\mathrm{pH}$ is the only variable of the test and the experiments were performed with constant concentration of other parameters as $20 \mathrm{mg} / \mathrm{l}$ dye concentration and $0.03 \mathrm{gr}$ of adsorbent for $30 \mathrm{~min}$. The result of experiment for removal of desired dye has been given in Figure 8. $\mathrm{pH}$ is highly effective in adsorption dosage. A lot of researches have been performed on the effect of $\mathrm{pH}$ on adsorption rate, which show the effects of different $\mathrm{pH}$ on the adsorption 
dosage. In acidic environments, hydrogen ions can act as competitors and reduce the uptake of positive ions. on the other hand, when the $\mathrm{pH}$ of the solution is increased too much, the positively charged amines increase, which reduces the adsorption of dye onto the adsorbent (GulbeyiDusum et al., 2005). As a result, acidic $\mathrm{pH}$ was selected as the optimal $\mathrm{pH}$.

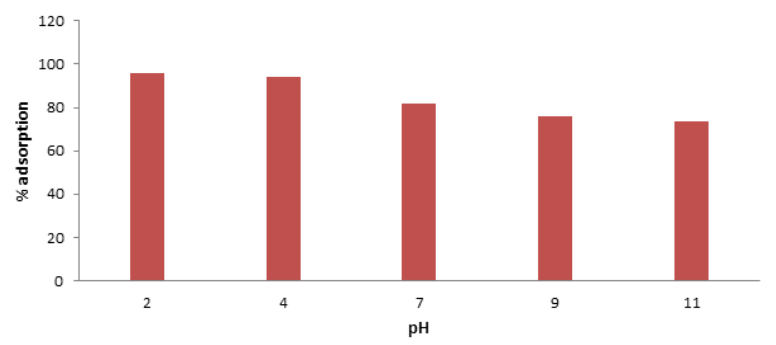

Figure 8. Effect of $\mathrm{pH}$ on the adsorption of Direct Black by CTS/SBA-15 (0.03 gr adsorbent, initial concentration: $20 \mathrm{mg} /$, contact time in $30 \mathrm{~min}$ )

\subsection{Effect of adsorbent dose}

The dependence of the adsorption of direct black ANBN dye on the amount of adsorbent in the range of 0.01 to 0.09 gr in $50 \mathrm{ml}$ of sample was investigated. In this case as in the previous steps the other optimized parameters were constant and the desired parameter was variable. Figure 9 shows the changes observed.According to the percentage of adsorption, an adsorbent dose of $0.05 \mathrm{gr}$ was selected as an optimal value.

\subsection{Effect of initial concentration}

The influence of this parameter on the adsorption efficiency was investigated in the ranging of $10-80 \mathrm{mg} / \mathrm{l}$ and the results have been given in Figure 10. Based on this, solutions with concentrations $10,20,40,60,80 \mathrm{mg} / \mathrm{l}$ of intended dye were prepared and $0.05 \mathrm{gr}$ of CTS/SBA-15 adsorbent dosage was added to that, after 30 min stirring, the adsorption rate of the filtered solutions was measured. From the results released, it can be stated that the optimal concentration is an initial $60 \mathrm{mg} / \mathrm{l}$ concentration. And at this concentration the adsorption has been performed with efficient percentage.

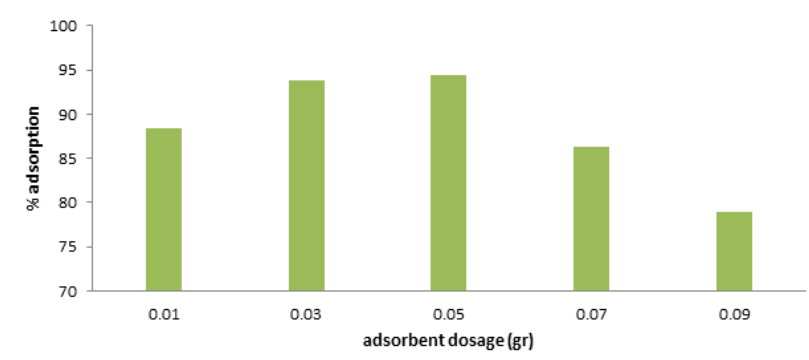

Figure 9. Effect of adsorbent dosage on the adsorption of Direct Black by CTS/SBA-15 ( $\mathrm{pH}=$ acidic, initial concentration: $20 \mathrm{mg} / \mathrm{l}$, contact time in $30 \mathrm{~min}$ )

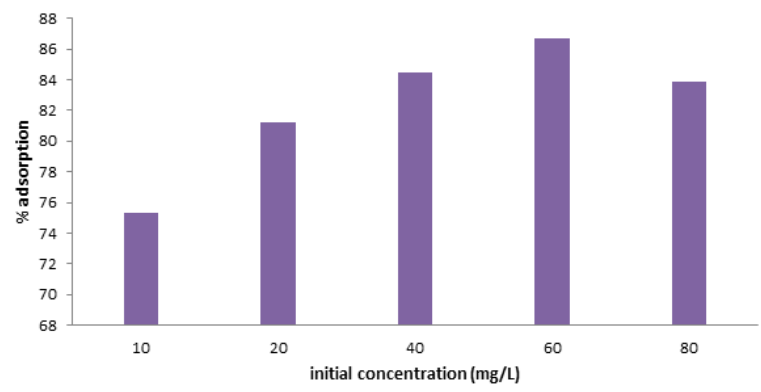

Figure 10. Effect of initial concentration on the adsorption of Direct Black by CTS/SBA-15 (0.05 gr adsorbent, $\mathrm{pH}=$ acidic, contact time in $30 \mathrm{~min}$ )

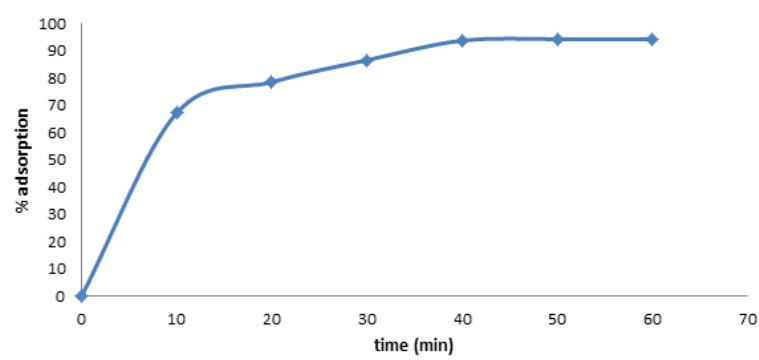

Figure 11. Effect of contact time on the adsorption of Direct Black by CTS/SBA-15 (0.05 gr adsorbent, $\mathrm{pH}=$ =acidic, initial concentration: $20 \mathrm{mg} / \mathrm{l}$ )

\subsection{Effect of contact time}

Contact time is one of other important factors influencing adsorption, which was studied in this research in range of 10 to $60 \mathrm{~min}$. For this aim, $60 \mathrm{mg} / \mathrm{l}$ of intended dye concentration was prepared and after adding $0.05 \mathrm{~g}$ of CTS/SBA-15 adsorbent to that, was put on the shaker for $10,20,30,40,50,60 \mathrm{~min}$ and after filtration, the adsorption dosage was measured. From the results given in Figure 11, it is released that the optimal contact time for this process is $40 \mathrm{~min}$.

\subsection{Comparison of direct black ANBN adsorption by CTS/SBA-15 and chitosan}

For better evaluation the performance of nanofiber (CTS/SBA-15), the adsorption of Direct Black ANBN was compared with CTS/SBA-15 and Chitosan (with the same condition), and the results are shown in Figures 12-15.

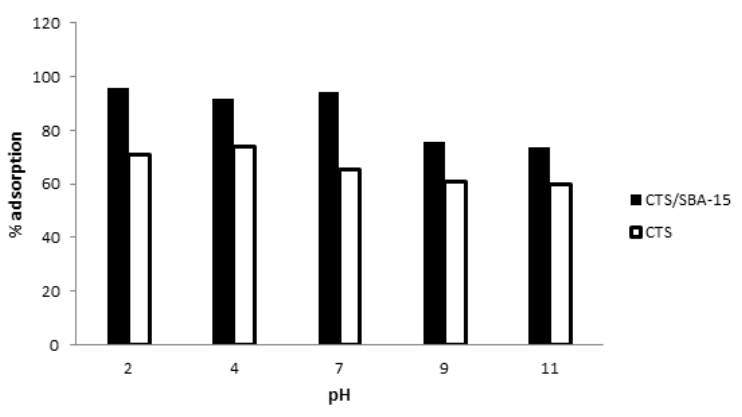

Figure 12. Effect of $\mathrm{pH}$ on the adsorption of Direct Black onto two different adsorbents: CTS/SBA-15 and Chitosan (0.03 gr adsorbent, initial concentration: $20 \mathrm{mg} / \mathrm{l}$, contact time in $30 \mathrm{~min}$ ) 


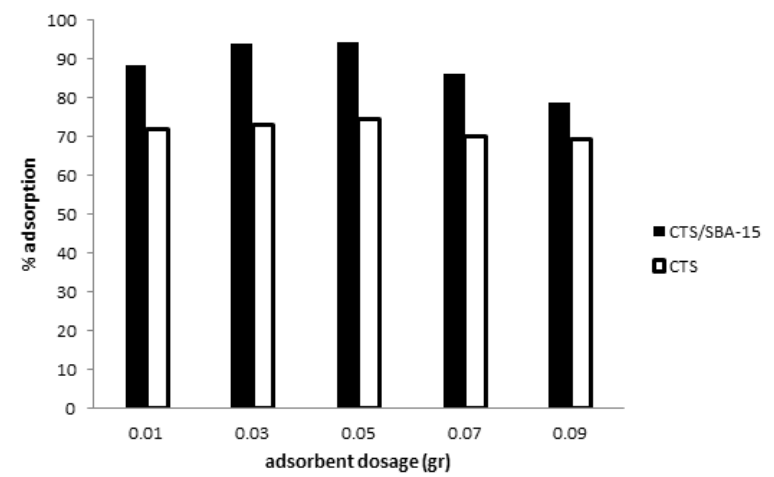

Figure 13. Effect of adsorbent dosage on the adsorption of Direct Black onto two different adsorbents: CTS/SBA-15 and Chitosan ( $\mathrm{pH}=$ acidic, initial concentration: $20 \mathrm{mg} / \mathrm{l}$, contact time in $30 \mathrm{~min}$ )

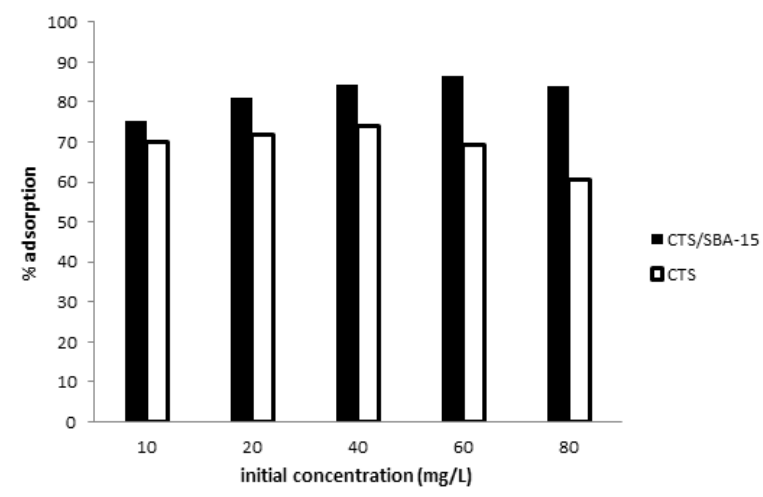

Figure 14. Effect of initial concentration on the adsorption of Direct Black onto two different adsorbents: CTS/SBA-15 and Chitosan ( $0.05 \mathrm{gr}$ adsorbent, $\mathrm{pH}=$ acidic, contact time in $30 \mathrm{~min}$ )

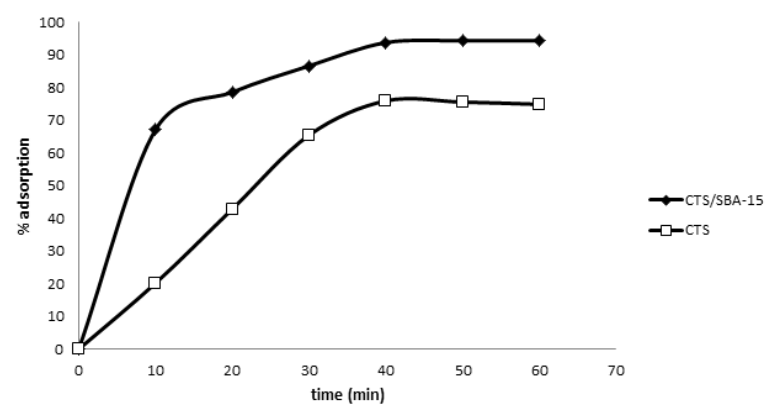

Figure 15. Evaluate effect of contact time on the adsorption of Direct Black onto two different adsorbents: CTS/SBA-15 and Chitosan ( 0.05 gr adsorbent, $\mathrm{pH}=$ acidic, initial concentration: 20 $\mathrm{mg} / \mathrm{l})$

\subsection{Adsorption isotherms study}

Measurements of equilibrium adsorption have been performed to determine the Maximum and ultimate capacity of adsorbent. The most common equations used are Langmuir and Freundlich isotherms (Lin et al., 2009).

\subsubsection{Freundlich equation}

The Freundlich isotherm is obtained by assuming a heterogeneous, multilayer surface with a non-uniform distribution of heat adsorption on the surface which is defined as following (Aliabadi et al., 2006).

$$
\log \left(\mathrm{q}_{\mathrm{e}}\right)=\log \left(\mathrm{K}_{\mathrm{f}}\right)+\frac{1}{\mathrm{n}} \log \left(\mathrm{C}_{\mathrm{e}}\right)
$$

In this equation $\mathrm{K}_{\mathrm{f}}$ and $\mathrm{n}$ are the constant rates of equation where $\log \mathrm{K}_{\mathrm{f}}$ is the width of the source and adsorption capacity indicator and $\mathrm{In}$ is the line slope and adsorption intensity indicator. The diagram of this isotherm for direct black ANBN dye is given in Figure 16.

As is clear from the figure, the amount of $\left(R^{2}=0.943\right)$ shows that adsorption of direct black ANBN dye on the CTS/SBA15 is fitted to Freundlich adsorption model (Table 2).

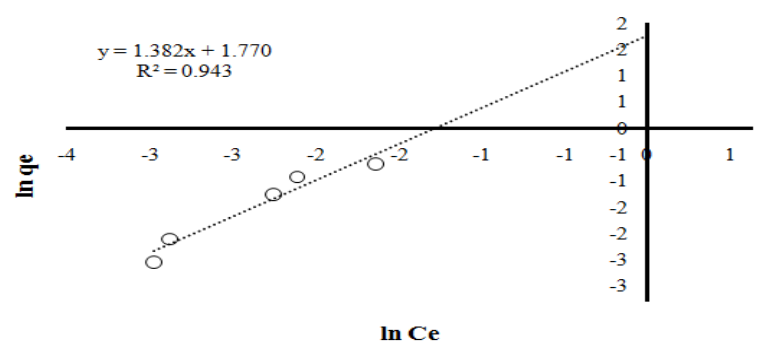

Figure 16. Freundlich adsorption isotherm for direct black dye adsorption by CTS/SBA-15

Table 2. Freundlich adsorption isotherm for direct black adsorption by CTS/SBA-15

\begin{tabular}{ccc}
\hline $\mathbf{R}^{\mathbf{2}}$ & $\mathbf{K}_{\mathbf{f}}$ & $\mathbf{N}$ \\
\hline 0.943 & 5.871 & 0.724 \\
\hline
\end{tabular}

\subsubsection{Langmuir equation}

This model includes assumptions including single layer adsorption, surface uniformity, and the removal of the interactions of the adsorbed molecules (Nadavala et al., 2009). The equation for Langmuir can be explained as following:

$$
\frac{\mathrm{C}_{\mathrm{e}}}{\mathrm{q}_{\mathrm{e}}}=\frac{1}{\mathrm{q}_{\mathrm{m}} \mathrm{b}}+\frac{\mathrm{C}_{\mathrm{e}}}{\mathrm{q}_{\mathrm{m}}}
$$

Where $C_{e}$ is the equilibrium concentration of dissolved substance, $q_{e}$ is the amount of dye adsorbed at equilibrium, $\mathrm{q}_{\mathrm{m}}$ is the maximum adsorption capacity and $\mathrm{b}$ is the constant rate of Langmuir model. Langmuir adsorption isotherm for direct black ANBN dye adsorption has been given in Figure 17. Considering the $q_{m}$ value (adsorption capacity of the adsorbent), it is found that the adsorbent CTS/SBA-15 have the relatively appropriate capacity for Direct Black ANBN adsorption (Table 3).

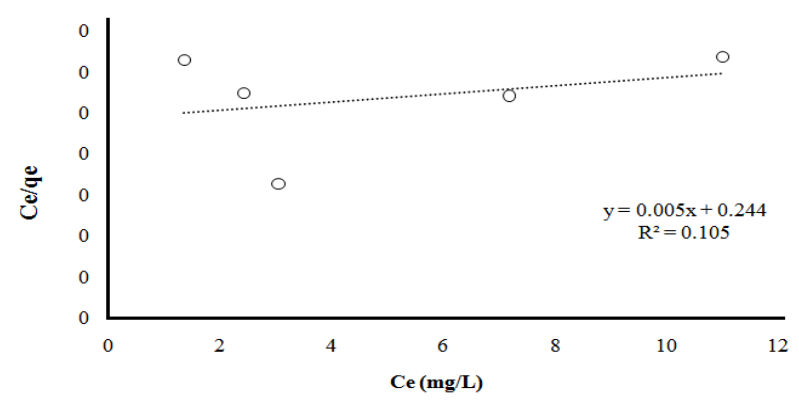

Figure 17. Langmuir adsorption isotherm for direct black dye adsorption by CTS/SBA-15 
Table 3. langmuir adsorption isotherm parameters for direct black dye adsorption by CTA/SBA-15

\begin{tabular}{ccc}
\hline $\mathbf{R}^{\mathbf{2}}$ & $\mathbf{q}_{\max }$ & $\mathbf{K}_{\mathbf{L}}$ \\
\hline .0105 & 200.000 & 0.020 \\
\hline
\end{tabular}

\section{Conclusion}

In this research, the productivity of chitosan-functionalized mesoporoussilica inelectrospinning method, was studied.The results obtained showed that removal of this dye from aqueous solutions by simple CTS/SBA-15 nanofibers in the acidic environment with $0.05 \mathrm{~g}$ adsorbent dosage, 40 min contact time, $60 \mathrm{mg} / \mathrm{l}$ initial concentration has the optimal and higher value. Also, removal of this dye is fitted to Freundlich adsorption isotherm. It is worth noting that the results are also achievable on a commercial scale. In addition, the removal of Direct Black ANBN by CTS/SBA-15 compared with Chitosan. According to the results, nanofiber (CTS/SBA-15) has better performance than its simple sample. According to the effects and toxicity of dye in the environment and also the better productivity of adsorbents used in this study rather than the other methods, it can be stated that using of synthesized CTS/SBA-15 nanofibers in electrospinning method is an appropriate replacement in removal of direct black ANBN dye from industrial wastewaters.

\section{Acknowledgements}

This essay is a part of Ph.D. dissertation of applied chemistry approved by Islamic Azad University Gorgan Branch. The authors are thankful for the assistance of University.

\section{References}

Alexa I.F., Ignat M. and Popovic R.F. (2012), In vitro controlled release of antihypertensive drugs intercalated into unmodified SBA-15 and MgO modified SBA-15 matrices, International Journal of Pharmaceutics, 436, 111-119.

Aliabadi M., Morshedzadeh K. and Soheyli H. (2006), Removal of hexavalent chromium from aqueous solution by lignocellulosic solid wastes, International Journal of Environmental Science and Technology, 3(3), 321-325.

Alireza B., Bahman H. and Reza R. (2014), Synthesis of nanoporous silica (SBA-15) functionalized with organic ligand including carboxylic acid and its application in pollutant removal of aquaculture industry, Journal of Applied Research in Chemistry, 3, 15-22, in Persian.

Almuhamed S., Khenoussi N., Bonne M., Schacher L., Lebeau B., Adolphe D. and Brendle J. (2014), Electrospinning of PAN nanofibers incorporating SBA-15-type ordered mesoporous silica particles, European Polymer Journal, 54, 71-78.

An A.K., Guo J., Lee E.-J., Jeong S., ZhaoY., Wang Z. and Leiknes T. (2017), PDMS/PVDF hybrid electrospun membrane with superhydrophobic property and drop impact dynamics for dyeing wastewater treatment using membrane distillation, The Journal of Membrane Science, 525, 57-67.

Badiei A.R., Laurent B., Nicolas C. and Mohammadi Ziarani Gh. (2006), Surface tailoring control in micelle templated silica, Journal of Organometallic Chemistry 691, 26, 5923-5931.

Bahrambeygi H., Sabetzadeh N., Rabbi A., Nasouri K., Shoushtari A.M. and Babaei M.R. (2013), The effect of polymer solution concentration on the structure of polyvinyl alcohol nanofibers, Journal of Polymer Research, 20, 74-84.
BoorboorAjdari F. and Behzad M. (2016), Efficient adsorption of $\mathrm{Cu}$ (II) and $\mathrm{Cr}$ (VI) metal ions by Schiff base modified SBA-15, Journal of Applied Chemistry, 37, 101-109.

Burleigh M.C., Dai S., Hagaman E.W. and Lin L.S. (2001), Imprinted polysilsesquioxanes for the enhanced recognition of metal ions, Chemistry of Materials, 13, 2537-2546.

Da'na E. and Sayari A. (2011), Adsorption of copper on aminefunctionalized SBA-15 prepared by co-condensation: Equilibrium properties, The Chemical Engineering Journal, 166, 445-453.

Devaraju S., Vengatesan M.R., Selvi M., Ashok Kumar A., Hamerton I., Go J.S. and Alagar M. (2013), Low surface free energy cyanate ester-silica hybrid (CE-SiO 2) nanomaterials for low k dielectric applications, RSC Advances, 3, 1291512921.

Erdem B., Erdem S., Çıtak A., Yavru C. and Öksüzoglu R.M. (2017), Preparation, Characterization and Catalytic Properties of SBA15-SO3H Supported Tungstophosphoric Acid for Esterification Reaction, Acta Physica Polonica A, 132, 1041-1044.

Garcia-Segura S., Dosta S., Guilemany J.M. and Brillas E. (2013), Solar photoelectrocatalytic degradation of Acid Orange 7 azo dye using a highly stable $\mathrm{TiO}_{2}$ photoanode synthesized by atmospheric plasma spray, Applied Catalysis B: Environmental, 132-133, 142-150.

GulbeyiDursun H.C. and Arzu Y. (2005), Adsorption of phenol from aqueous solution by using carbonised beet pulp, Journal of Hazardous Materials, B125, 175-182.

Jiang Y., Sun Y., Liu H., Zho F. and Yin H. (2008), Solar photocatalytic decolorization of $\mathrm{Cl}$ Basic Blue 41 in an aqueous suspension of $\mathrm{TiO}_{2}-\mathrm{ZnO}$, Dyes Pigments, 78, 77-83.

Kaur A., Vats S., Rekhi S., Bhardwaj A., Goel J., Tanwar R.S. and Gaur K.K. (2010), Physico-chemical analysis of the industrial effluents and their impact on the soil microflora, Procedia Environmental Sciences, 2, 595-599.

Khataee A.R, Zarei M. and Dehghan G. (2011), Biotreatment of a triphenylmethane dye solution using a Xanthophyta alga: Modeling of key factors by neural network, Journal of the Taiwan Institute of Chemical Engineers, 42, 380-86.

Lin S.H. and Juang R.S. (2009), Adsorption of phenol and its derivatives from water using synthetic resins and low-cost natural adsorbents: a review, Journal of Environmental Management, 90(3), 1336-1349.

Liversidge R., Lloyd G., Wase D. and Forster C. (1997), Removal of Basic Blue 41 dye from aqueous solution by linseed cake, Process Biochemistry, 32(6), 473-477.

Munusamy S. and Apama R.S.L. (2013), Photocatalytic effect of TiO 2 and the effect of dopants on degradation of brilliant green, Sustainable Chemical Processes, 1, 4-12.

Nadavala S.K. (2009), Biosorption of phenol and o-chlorophenol from aqueous solutions on to chitosan-calcium alginate blended beads, Journal of Hazardous Materials, 162(1), 482489.

Nasouri K., Bahrambeygi H., Rabbi A., Shoushtari A.M. and Kaflou A. (2012), Modeling and optimization of electrospun PAN nanofiber diameter using response surface methodology and artificial neural networks, Applied Polymer Science, 126, 127135.

Nasouri K., Shoushtari A.M., Kaflou A., Bahrambeygi H. and Rabbi A. (2012), Single-wall carbon nanotubes dispersion behavior and its effects on the morphological and mechanical 
properties of the electrospun nanofibers, Polymer Composites, 33, 1951-1959.

Nilsson I., Moller A., Mattiasson B., Rubindamayugi M. and Welander U. (2006), Decolorization of synthetic and real textile wastewater by the use of white-rot fungi, Enzyme and Microbial Technology, 38(1-2), 94-100.

Nunez L., García-ortal J.A. and Torrades F. (2007), Study of kinetic parameters related to the decolourization and mineralization of reactive dyes from textile dyeing using Fenton and photo-Fenton processes, DyesPigments, 75, 647-52.

Ohima I., Luke N.O. and Amraibure O. (2009), Studied on the pollution potential of wastewater from textile processing factorises in Kaduna, Nigeria, The Journal of Toxicology and Environmental Health, 1, 34-37.

Rabbi A., Nasouri K., Bahrambeygi H., Shoushtari A.M. and Babaei M.R. (2012), RSM and ANN approaches for modeling and optimizing of electrospun polyurethane nanofibers morphology, Fibers and Polymers, 13, 1007-1014.

Saeed S.M., Zandi M. and Mirzadeh H. (2012), Effect of Solution Surface Tension on Morphology of PLGA and Gelatin Electrospun Fibers, Iranian Journal of Polymer Science and Technology, 25, 3-10.

Sarioglu (Cebeci) M. and Askal M. (2017), Biosorption of Azo Dye (Maxilon Red and Everzol Red) on to natural and modified waste sludge, Global NEST Journal, 20, 25-3.

Shokoohi R., Torkshavand Z., Bajalan S., Zolghadnasab H. and Khodayari Z. (2018), Efficient phenol removal from aqueous solution using iron-coated pumice and leca as an available adsorbents: evalution of kinetics and isotherm studies, Global NEST Journal, 21, 91-97.

Ullah R., Atilham M., Aparicio S., Canlier A. and Yavuz C.T. (2015), Insights of $\mathrm{CO} 2$ adsorption performance of amine impregnated mesoporous silica (SBA-15) at wide range pressure and temperature conditions, International Journal of Greenhouse Gas Control, 43, 22-32.

Uysal Y. and Bilgic M. (2018), Color removal from wastewater by using two-step (biological and chemical) aerobic filter reactors, Global NEST Journal, 20, 7-13.

Yue Z.H., Ru J., Jiang G.Y. and Qian F.Y. (2010), Effect of key operational factors on decolorization of methyl orange during $\mathrm{H}_{2} \mathrm{O}_{2}$ assisted $\mathrm{CdS} / \mathrm{TiO}_{2} /$ ploymer nanocomposite thin films under simulated solar light irradiation, Separation and Purification Technology, 74, 187-94.

Zhao D., Feng J., Huo Q., Melosh N., Fredrckson G.H., Chmelka B.F. and Stucky G.D. (1998), Triblock Copolymer Syntheses of Mesoporous Silica with Periodic 50 to 300 Angstrom Pores, Science, 279(5350), 548-552.

Zhao D., Huo Q., Feng J., Chmelka B.F. and Stucky G.D. (1998), Nonionic Triblock and Star Diblock Copolymer and Oligomeric Surfactant Syntheses of Highly Ordered, Hydrothermally Stable, Mesoporous Silica Structures, Journal of the American Chemical Society, 120(24), 6024-6036.

Zhao W., Song B., Tao J., Zhang J., Huang M. and Gou M. (2016), Preparation of $\mathrm{TiO}_{2}$ /activated carbon composites for photocatalytic degradation of RhB under UV light irradiation, Journal of Nanomaterials, 2016, 1-10. 